\title{
Molecular mechanisms underlying the mitosis-meiosis decision
}

\author{
Yuriko Harigaya \& Masayuki Yamamoto* \\ Department of Biophysics and Biochemistry, Graduate School of Science, University of Tokyo, 7-3-1 Hongo, \\ Bunkyo-ku, Tokyo, 113-0033, Japan; Tel: +81-35-8414386; Fax: +81-35-802-2042; \\ E-mail:yamamoto@biochem.s.u-tokyo.ac.jp \\ * Correspondence
}

Key words: cell cycle regulation, fission yeast, mRNA elimination, nutrition, pheromone signaling, RNA-binding protein

\begin{abstract}
Most eukaryotic cells possess genetic potential to perform meiosis, but the vast majority of them never initiate it. The entry to meiosis is strictly regulated by developmental and environmental conditions, which vary significantly from species to species. Molecular mechanisms underlying the mitosis-meiosis decision are unclear in most organisms, except for a few model systems including fission yeast Schizosaccharomyces pombe. Nutrient limitation is a cue to the entry into meiosis in this microbe. Signals from nutrients converge on the activity of Mei2 protein, which plays pivotal roles in both induction and progression of meiosis. Here we outline the current knowledge of how a set of environmental stimuli eventually activates Mei2, and discuss how Mei2 governs the meiotic program molecularly, especially focusing on a recent finding that Mei2 antagonizes selective elimination of meiotic messenger RNAs.
\end{abstract}

\section{Introduction}

Meiosis is a process for forming haploid gametes from diploid germ cells, which is essential for sexually reproducing species to transmit genetic information to the next generation. Meiosis drives a specialized cell cycle that consists of one round of DNA synthesis followed by two successive rounds of $\mathrm{M}$ phase. This procedure, which halves the genetic material, seems to be highly conserved in eukaryotes. However, cues that cause cells to enter the meiotic pathway appear to vary greatly among species. In multicellular organisms, extrinsic cues from surrounding cells control the differentiation of germline stem cells that will enter the meiotic cell cycle. In lower eukaryotes such as budding yeast Saccharomyces cerevisiae or fission yeast Schizosaccharomyces pombe, a reduction of available nutrition in the environment triggers the entry into the meiotic cell cycle. In either case, the decision to enter the meiotic cell cycle is tightly regulated in order to prevent execution of the meiotic program in inappropriate developmental contexts. Here, we discuss controls to commit cells to meiosis in fission yeast, which is an excellent model system for study of the mitosis-meiosis decision. Our discussion focuses on the regulatory pathways underlying the two types of cell cycle programs.

Meiotic differentiation includes complex sequential events. Yeast meiosis may be conventionally divided into two parts: (1) exit from the mitotic cell cycle and (2) induction of the alternate, meiotic cell cycle program, in which mitotic events may occur in a peculiar manner and order. The former process in fission yeast has been characterized as a multilayered network of positive and negative factors, which are 
regulated at various levels of gene expression from transcription initiation to protein modification and degradation (Yamamoto 1996, 2004, Yamamoto et al. 1997). The network also involves many feedback loops to intensify the commitment to meiosis, which may ensure that mitosis and meiosis become mutually exclusive. In contrast, the latter process has been less characterized, although it is generally assumed to be achieved by a highly coordinated transcriptional induction of numerous genes that are specifically required for meiosis (Mata et al. 2002). The meiosis-specific gene products are likely to be required (1) to modify the basic cell duplication machinery that operates in the mitotic cell cycle, so that DNA synthesis followed by two successive nuclear divisions will be ensured and (2) to reorganize the cell morphology, which results in the formation of asci containing four haploid spores, the counterparts of gametes in higher eukaryotes. In the next four sections we will describe the developmental fates available for fission yeast cells and overview the current knowledge of cell cycle regulation and gene expression during mitosis and meiosis in this microbe. We will then look at key factors that constitute the central regulatory module for meiosis in fission yeast, namely the Pat1-Mei2 system, focusing on how the exit from the mitotic cell cycle and the initiation of the meiotic program are governed by this system. Finally, we will address how the environmental cues convey a signal to the key factors and regulate the exit from the mitotic cell cycle.

\section{Developmental fates of fission yeast}

Under conditions rich in nutrition, fission yeast cells proliferate through mitotic cell cycles, mainly as haploids, which carry one of the two mating types denoted as $h^{+}(P)$ and $h^{-}(M)$. As in higher eukaryotes, the mitotic cell cycle can be divided into four phases, namely $\mathrm{G}_{1}, \mathrm{~S}, \mathrm{G}_{2}$, and M. Mitotic cells assume one of the three alternative fates according to the environmental conditions: They may (1) continue to progress through the mitotic cell cycle, (2) enter into the quiescent stationary phase, or (3) differentiate into the conjugation/meiosis pathway. Fission yeast cells can enter the stationary phase from either mitotic $G_{1}$ or $G_{2}$. Different environmental stimuli impose different effects on the mitotic cell cycle.
Glucose starvation, for example, primarily arrests proliferating cells in $\mathrm{G}_{2}$ and lead them to the stationary phase (Costello et al. 1986). Under nitrogen starvation, in contrast, haploid cells arrest in $G_{1}$ and enter the stationary phase, usually after performing two or three rounds of rapid mitotic cycle. If cells of the opposite mating type are neighboring under nitrogen starvation, haploid cells mate to form zygotes, which subsequently undergo meiosis and generate four haploid spores. Importantly, only haploid cells in $\mathrm{G}_{1}$ phase can initiate the conjugation process. Zygotes can grow as diploids if they are transferred to a rich medium immediately after conjugation. These diploid cells undergo meiosis and form spores when they exhaust the available nutrients. Whether they are zygotes or diploids, meiotic cells follow essentially the same pathway. They arrest transiently in $G_{1}$, initiate one round of DNA synthesis, and perform two consecutive nuclear divisions, called first and second meiotic divisions (alternatively, meiosis I and meiosis II). Importantly also, only $\mathrm{G}_{1}$-arrested diploid cells can enter the meiotic pathway.

\section{Cell cycle regulation during mitosis}

During the mitotic cell cycle, the onset of $\mathrm{S}$ phase and that of $\mathrm{M}$ phase are under tight regulation, in order to ensure that these two phases are coupled in the correct order (reviewed in MacNeill \& Nurse 1997, Moser \& Russell 2000). The major player in this control is $\mathrm{Cdc} 2$, the cyclin-dependent kinase (CDK), whose activity is low in $\mathrm{G}_{1}$ phase, moderate during $S$ phase and $G_{2}$ phase, and high during $M$ phase. Cdc2 is associated with cyclin $\mathrm{Cig} 2$ at the $\mathrm{G}_{1} / \mathrm{S}$ transition (Bueno \& Russell 1993, Connolly \& Beach 1994, Obara-Ishihara \& Okayama 1994). Deletion of cig2 delays entry to $\mathrm{S}$ phase, but does not completely block the transition, because mitotic cyclin Cdc13 can eventually substitute the function of Cig2 (Fisher \& Nurse 1996). The entry to S phase also requires the function of $\mathrm{Cdc10}$, which constitutes a transcription factor complex called DSC1 (Lowndes et al. 1992). The DSC1 complex is thought to contain Cdc10, Res1, Res2, and Rep2 to mediate $\mathrm{G}_{1} / \mathrm{S}$ specific transcription through both stimulatory and repressive functions (Tanaka et al. 1992: Caligiuri \& Beach 1993, Miyamoto et al. 1994, 
Zhu et al. 1994, Nakashima et al. 1995, Baum et al. 1997, Whitehall et al. 1999). The DSC1 targets include genes essential for DNA replication, such as $c d c 18$ encoding a fission yeast homolog of conserved replication factor Cdc6, cdtl encoding another conserved replication factor, and $c d c 22$ encoding ribonucleotide reductase (Kelly et al. 1993, FernandezSarabia et al. 1993, Hofmann \& Beach 1994, White et al. 2001). The onset of mitosis is driven by the Cdc2-Cdc13 complex, which is regulated positively by the Cdc25 phosphatase and negatively by the Wee1 and Mik1 kinase (Lundgren et al. 1991, Millar et al. 1991). E3 ubiquitin ligase APC/C (anaphasepromoting complex/cyclosome) associated with its activator Slp1 (APC/C $\left.\mathrm{C}^{\mathrm{Slp} 1}\right)$ is required for $\mathrm{Cdc} 13 \mathrm{deg}$ radation upon exit from $\mathrm{M}$ phase, whereas $\mathrm{APC} / \mathrm{C}$ associated with another activator Ste9 $\left(\mathrm{APC} / \mathrm{C}^{\mathrm{Ste} 9}\right)$ and the CDK inhibitor (CKI) Rum1 are responsible for suppressing $\mathrm{Cdc} 2$ activity during $\mathrm{G}_{1}$ phase (Correa-Bordes \& Nurse 1995, Yamaguchi et al. 1997, Kitamura et al. 1998, Blanco et al. 2000).

An important concept in the yeast cell cycle is 'Start', which is a point (or an interval) during $\mathrm{G}_{1}$ at which the cell becomes committed to the mitotic cell cycle. Prior to passing Start, cells have the potential to take the three alternative developmental programs mentioned above. At Start, cells carefully monitor their own size and nutritional conditions and determine their fate. The transition at $\mathrm{G}_{2} / \mathrm{M}$ is another control point in the mitotic cell cycle, where again both size and nutritional controls are in operation. Cell cycle regulators relevant to the switch between the mitotic and meiotic cell cycles are shown schematically in Figure 1. Regulation by TOR complex TORC1, included in this figure, will be discussed at the end of this review.

\section{Cell cycle regulation during meiosis}

It has been suggested that $\mathrm{Cdc} 2$ kinase plays essential roles in driving the meiotic cell cycle. Genetic analyses have established the absolute requirement of the $c d c 2$ gene for the meiotic $\mathrm{G}_{1} / \mathrm{S}$ transition and the second meiotic division (Iino et al. 1995). Cdc2 cooperates with cyclin Cig2 to launch premeiotic $\mathrm{S}$ phase (Borgne et al. 2002). Cyclin Cdc13 and the CDK activator Cdc25 are required for both the first and second meiotic divisions (Iino et al. 1995)
(Figure 1). During premeiotic $\mathrm{S}$ phase, transcription of two groups of genes are simultaneously activated: (1) a group that is also expressed in the mitotic $\mathrm{G}_{1} / \mathrm{S}$ interval and required for DNA synthesis (e.g., $c d c 18$, $c d t 1$, and $c d c 22$ ) and (2) a group that is specific to meiosis, including the rec genes (e.g., rec8 and recll) involved in meiotic recombination (Fox \& Smith 1998, Watanabe et al. 2001, Mata et al. 2002). Genes of both groups appear to be regulated by the DSC1 complex, which is responsible for the transcriptional activation of the former class of genes in mitotic cells (White et al. 2001, Cunliffe et al. 2004). It is proposed that a meiotic form of DSC1, containing Cdc10, Res2, and a meiosis-specific component Rep1, is responsible for the meiotic pattern of gene expression (Sugiyama et al. 1994). The meiosis-specific S phase transcription may lead to a peculiar mode of DNA synthesis coupled with incorporation of a meiosis-specific cohesin complex (Watanabe et al. 2001) and recombination at a high frequency, eventually followed by two consecutive nuclear divisions.

Classical genetic analyses have identified meiotic mutants that arrest at a specific point in the meiotic cell cycle (reviewed in Yamamoto et al. 1997). The mei2, mei3, and mei4 mutants arrest with a single nucleus in the cell, whereas the mesl mutants arrest with two nuclei, indicating cell cycle arrest before the second meiotic division. The mei2 and mei3 mutants fail to proceed to premeiotic $\mathrm{S}$ phase, whereas the mei4 mutant arrests before the first meiotic division after completing DNA synthesis. One possible function of these gene products is to act on cell cycle machineries that are utilized also in the mitotic cell cycle. Indeed, emerging evidence supports this idea with regard to mei4 and mes1. mei4 encodes a transcription factor that is required for transcriptional activation of $c d c 25$ during meiosis, in addition to numerous other target genes (Iino et al. 1995, Horie et al. 1998, Mata et al. 2002) (Figure 1). Lowered Cdc25 activity presumably explains the cell cycle arrest in the mei4 mutant, because artificial expression of $c d c 25$ restores meiotic divisions in the mei4A cells (H. Murakami, personal communication). Mes1 binds and inhibits the APC/C activator Slp1, and hence secures the Cdc2-Cdc13 MPF activity to carry out the second meiotic division (Izawa et al. 2005). Meanwhile, mei3 is required to activate the mei2 gene product, Mei2, which is the 


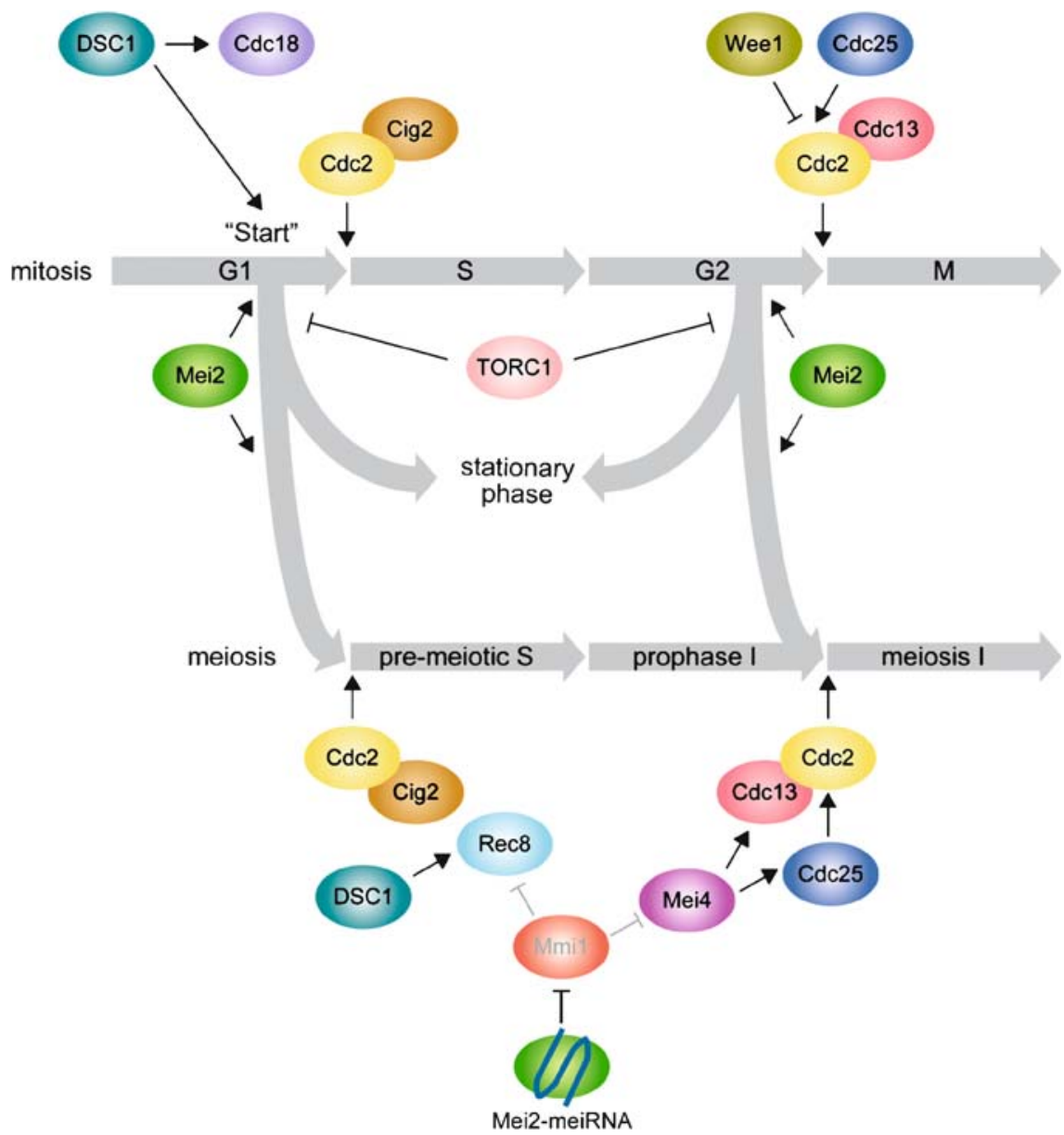

Figure 1. A simplified network of cell cycle regulators operating at the mitosis-meiosis decision in fission yeast. The upper line represents the mitotic cycle, and the lower, the meiotic pathway up to meiosis I. An arrow indicates each cell-cycle window but its length is arbitrary and does not necessarily represent the time span of the window. With regard to the cell cycle switch, active TOR complex TORC1 suppresses exit from the mitotic cell cycle (discussed in the text), whereas Mei2 functions to promote it. Upon exit from the mitotic cycle, Mei2 plays an essential role for execution of the alternate meiotic cell cycle program. To initiate premeiotic S phase, the DSC1 complex promotes transcription of two groups of genes: (1) genes required for DNA replication per se, and (2) genes that function specifically for meiotic replication, such as rec8. A subpopulation of the Mei2 protein binds to meiRNA and form a single nuclear dot, which sequesters Mmil. Mmil eliminates a subset of meiosis-specific transcripts (e.g., mei4 and rec8) during the mitotic cell cycle, to suppress the incidence of untimely meiosis (more details in Figure 2). The sequestration of Mmi1 by the Mei2 dot is apparently essential for the promotion of meiosis I. Mei2 normally induces meiosis only from $\mathrm{G}_{1}$ phase of the mitotic cell cycle, but under artificial conditions it can induce meiosis from $\mathrm{G} 2$ phase.

master regulator of meiosis in fission yeast (see below). The molecular function of Mei2 has remained largely unknown until recently, and will be discussed extensively in this review.

\section{Expression and selective elimination of meiotic mRNAs}

The cell cycle switch from mitosis to meiosis is accompanied by a striking change in gene expression profiles. Many genes required for the progression of meiosis are up-regulated by a series of transcription factors (Mata et al. 2002). In addition, our recent study has revealed a new mode of regulation that contributes to the differential gene expression between mitosis and meiosis (Harigaya et al. 2006), as outlined below.

This new regulation operates during the mitotic cell cycle to suppress meiotic gene expression. A hint of the presence of this kind of regulation came from an observation that some meiosis-specific transcripts 
a

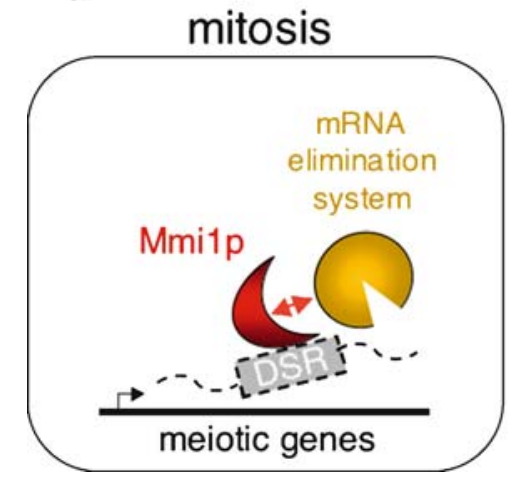

b

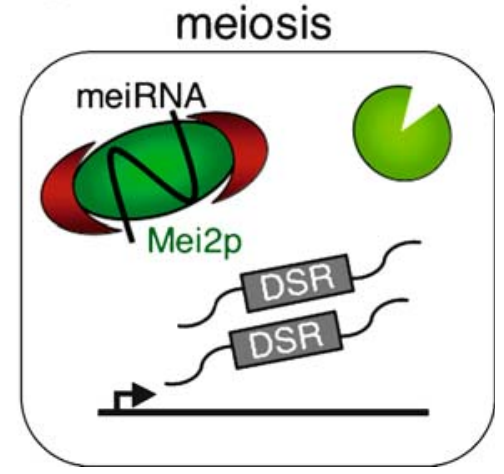

Figure 2. Selective elimination of meiosis-specific mRNAs in mitotic cells. (a) A number of meiosis-specific transcripts in fission yeast carry a region designated DSR, which renders them eliminated if they are expressed during the mitotic cell cycle. Mmi1, a YTH-family RNAbinding protein localized in the nucleus, binds to the DSR and promotes elimination of the transcript, probably cooperating with RNAdegrading exosome machinery. (b) During meiotic prophase, Mei2, which has affinity for Mmi1, forms a dot structure in the nucleus together with meiRNA and sequesters Mmil to this dot, so that meiosis-specific transcripts become free from Mmi1-dependent mRNA elimination and remain stably. This figure is reproduced from the Supplemental Figure S1 of our previous publication (Harigaya et al. 2006).

failed to accumulate in mitotic cells even when they were transcribed from a constitutive promoter. These transcripts include mei4 mRNA, which encodes a key transcription factor (Horie et al. 1998), and rec8 mRNA, which encodes a subunit of the meiotic cohesin complex (Watanabe \& Nurse 1999). They carry a cis-acting region termed DSR (determinant of selective removal), which is responsible for the block of their accumulation in mitotic cells (Harigaya et al. 2006). Mmi1, a YTH-family protein (Stoilov et al. 2002), binds DSR RNA and causes removal of DSRcontaining transcripts. Moreover, Rrp6, a fission yeast ortholog of a conserved nuclear-restricted component of the exosome (Houseley et al. 2006), seems to be involved in this removal, suggesting the possibility that DSR-containing transcripts are selectively degraded in the nucleus of growing cells (Harigaya et al. 2006) (Figure 2a). Importantly, inactivation of mmil leads to a severe growth defect due to untimely expression of the meit gene and genes stimulated by Mei4. This indicates that the DSR-dependent elimination of meiosis-specific transcripts is not a mere safeguard mechanism that would operate when their promoter happens to be accidentally activated; Rather, dividing cells should keep this system constantly in operation in order to curtail expression of meiotic genes. Microarray analyses have shown that at least a dozen meiosis-specific transcripts are subject to elimination by the DSR-Mmi1 system (Harigaya et al. 2006). It seems unlikely, however, that the DSR-Mmi1 system targets all the genes up-regulated during meiosis. For example, rec12, which encodes an ortholog of the conserved meiotic DSB inducer Spo11 (Keeney et al. 1997), does not appear to be regulated by this system (our unpublished results).

Fission yeast cells may use another mechanism to prevent expression and function of meiotic genes during proliferation. It has been found that a fraction of intron-containing transcripts remain unspliced until a specific period during meiosis, among which are crsl and reml mRNAs, both encoding meiosisspecific cyclin (Averbeck et al. 2005). Analyses of gene chimeras have indicated that splicing of $\mathrm{crs} 1$ and reml mRNAs is prevented in mitotically growing cells through sequences located outside the coding region (Averbeck et al. 2005). During meiosis, the inhibition of reml splicing appears to be overcome by the function of Mei4 (Malapeira et al. 2005).

\section{The Pat1-Mei2 system: central regulator for fission yeast meiosis}

Discovery of the patl mutation (initially also called ranl) opened the door to molecular understanding of the meiotic entry in fission yeast (Beach et al. 1985, Iino \& Yamamoto 1985, Nurse 1985). Cells carrying the pat1-114 mutation show temperature-sensitive growth and perform ectopic meiosis and sporulation at the restrictive temperature, regardless of nutritional 
conditions and the ploidy of the cells, indicating that Pat1 is a factor that prevents cells from entering meiosis when they should grow mitotically.

The patl gene encodes a Ser/Thr protein kinase (McLeod \& Beach 1988), which phosphorylates Mei2, an RRM-type RNA-binding protein critical for the entry to meiosis, on residues Ser438 and Thr527 (Watanabe et al. 1997). Even weak expression of mutant Mei2 carrying alanine in these two positions (Mei2 ${ }^{\mathrm{SATA}}$ ) results in ectopic meiosis and sporulation, as does inactivation of Pat1 kinase (Watanabe et al. 1997). This indicates that Mei2 is a critical target of Pat 1 and a pivotal factor for the entry to meiosis.

How is the Mei2 activity suppressed through phosphorylation by Pat1 kinase? As one possible mechanism, this phosphorylation confers a shorter half-life to Mei2, due to increased susceptibility of it to proteasome-dependent destruction involving E2 Ubc2 and E3 Ubr1 (Kitamura et al. 2001). As another possibility, phosphorylated Mei2 shows increased affinity for $\operatorname{Rad} 24$, the major 14-3-3 protein in fission yeast. The binding of $\operatorname{Rad} 24$ to phosphorylated Mei2 inhibits association of Mei2 with meiRNA (Sato et al. 2002), which is a noncoding RNA that cooperates with Mei2 to propel meiotic progression (Watanabe \& Yamamoto 1994). Deletion of rad24 accelerates commitment to meiosis, but this mutation by itself does not result in such a conspicuous meiosis-inducing phenotype as shown by the pat1-114 or mei2 $2^{\text {SATA }}$ mutation. Thus, it seems that there are yet-unidentified functions of Mei2 that are critical to inducing meiosis and are inhibited by the phosphorylation by Pat 1 .

\section{How could Mei2 provoke and govern the meiotic program?}

Activation of Mei2 can confer a cell cycle switch from mitosis to meiosis. Therefore, understanding the molecular function of Mei2 is a key to fission yeast meiosis, but this has not been an easy task. In addition to its function as a meiotic inducer, Mei2 is known to play essential roles in the induction of premeiotic $\mathrm{S}$ phase and first meiotic division. This has been deduced from the following observations. (1) Cells devoid of the mei2 gene can cease growth in response to nutritional starvation but cannot initiate premeiotic DNA synthesis. (2) In a temperature- sensitive mutant of mei2 (mei2-33), the meiotic cell cycle is arrested before the initiation of first meiotic division after completing premeiotic DNA synthesis (Watanabe \& Yamamoto 1994). An early study has shown that transcription of mei2 is up-regulated by Ste11, an HMG-type transcription factor, upon nutrient deprivation (Sugimoto et al. 1991). Mei2 has an intrinsic feature to shuttle between the nucleus and the cytoplasm (Sato et al. 2001). Upon entry to meiosis, a subpopulation of this protein forms a peculiar single dot in the nucleus, which we hereafter call the Mei2 dot (Watanabe et al. 1997, Yamashita et al. 1998). The Mei2 dot is located on the chromosomal locus of the sme 2 gene, from which meiRNA is transcribed (Shimada et al. 2003). In sme2A cells, which lack meiRNA, the Mei2 dot formation is impaired, and the meiotic cell cycle is blocked prior to the initiation of meiosis I (Watanabe et al. 1997, Yamashita et al. 1998). Thus, meiRNA performs a function required specifically for meiosis I, in cooperation with Mei2. Given that mei4 mRNA is undetectable in sme2 $\Delta$ cells (A. Yamashita and M. Yamamoto, unpublished), the meiotic cell cycle arrest in this mutant appears to be attributable at least in part to the absence of Mei4 function.

In summary, Mei2 is apparently involved in multiple steps of sexual differentiation: (1) the withdrawal from the mitotic division cycle; (2) the start of premeiotic S phase; and (3) the initiation of meiosis I. It has been speculated that the molecular activity of Mei2 could be linked to the cell cycle regulators and gene expression machinery, either directly or indirectly. Indeed, our recent study has demonstrated that one function of Mei2 is to act on a regulatory factor for meiotic gene expression. We will discuss this in the next section.

\section{Mei2 turns off the DSR-Mmi1 system}

Our study has revealed a direct link between Mei2 and the DSR-Mmi1 system for elimination of meiosis-specific transcripts (Harigaya et al. 2006). This was recognized originally through localization analysis of Mmi1. During vegetative growth, Mmi1 was localized to multiple foci in the nucleus, but it converged to a single dot that overlapped with the Mei2 dot during meiotic prophase. The concentration of Mmi1 did not occur in the absence of the Mei2 dot in mei2 $\Delta$ or sme $2 \Delta$ cells. These observations have 
lead us to speculate that Mei2 may turn off the DSR-Mmi1 system by sequestering Mmi1 to the dot, and thereby ensure stable expression of meiosisspecific transcripts (Figure 2b). Consistent with this idea, the arrest prior to meiosis I caused by loss of the Mei2 dot can be rescued by a reduction in the Mmil activity, presumably because it allows accumulation of DSR-containing transcripts, mei4 mRNA among others (Harigaya et al. 2006).

Shutdown of the DSR-Mmi1 system by the Mei2 dot can fully account for the function of Mei2 necessary to initiate meiosis I. However, it does not appear to be the only task that Mei2 executes during meiosis. This is because inactivation of Mmil does not lead to the conspicuous meiosis-inducing phenotype of the mei2 $2^{S A T A}$ mutant. Rather, loss of mmil causes a severe growth defect that seems likely to be a perturbation of the mitotic cell cycle by untimely function of meiotic genes (our unpublished results). This may also indicate that Mei2-dependent inactivation of the DSR-Mmil system should be under strict temporal control so that expression of meiotic genes may never precede the withdrawal from the mitotic cycle. There may be a mechanism that signals Mei2, after the exit from the mitotic cycle, to launch the next action to carry forward the meiotic program by promoting expression of DSR-containing transcripts.

\section{Mei2 and growth inhibition}

Transient $\mathrm{G}_{1}$ arrest occurs at the very beginning of both patl-driven and mei2 $2^{\text {SATA }}$-driven meiosis. Although mei2 $\Delta$ diploids can arrest in $\mathrm{G}_{1}$ in response to nutritional starvation, the kinetics are delayed (Watanabe \& Yamamoto 1994). Loss of mei2 function completely blocks patl-driven meiosis. Furthermore, the pat1-114 mei2s double mutant can continue to grow at the temperature restrictive for pat1-114. All these observations indicate a function of active Mei2 to inhibit growth and cause transient arrest in $G_{1}$ phase at the entry to the meiotic cell cycle. The molecular mechanism by which unphosphorylated Mei2 induces transient $G_{1}$ arrest remains elusive. It is possible that a reduction in the level of rep2 mRNA in response to the expression of mei2 $2^{\text {SATA }}$ may contribute to the cell cycle arrest in $\mathrm{G}_{1}$, given that deletion of rep2 leads to the accumulation of cells in $\mathrm{G}_{1}$ (Nakashima et al. 1995,
Watanabe et al. 1997). The link between Mei2 and the Cdc2 activity, whose down-regulation is essential for imposing cell cycle arrest, has not been pursued thoroughly but it may be indirect (see below).

\section{Mei2 and meiotic $G_{1} / S$ transition}

The function of Mei2 is absolutely necessary for the meiotic $\mathrm{G}_{1} / \mathrm{S}$ transition (or 'Start' of the meiotic cell cycle), since mei2 $\Delta$ diploid cells starved of nitrogen are unable to increase the DNA content after arresting at $\mathrm{G}_{1}$ (Watanabe \& Yamamoto 1994). The molecular mechanism by which Mei2 promotes DNA synthesis also remains elusive. Given that Mei2 $2^{\text {SATA }}$ induces a transcriptional switch between repl and rep2 (Watanabe et al. 1997), and that transcription of rep1, the putative meiotic counterpart of rep2, is essential for premeiotic S phase (Sugiyama et al. 1994), it is possible that Mei2 may promote initiation of premeiotic S phase by enhancing repl expression. It should be noted, however, that repl mRNA can be expressed in mei2 $\Delta$ diploid cells in response to nitrogen starvation (Sugiyama et al. 1994). Nevertheless, it may be presumed that the protein level and/or the activity of Rep1 may be reduced in the absence of mei2 and cannot support premeiotic DNA synthesis. Cdc2 is essential for premeiotic DNA synthesis and is thought to form a complex with cyclin Cig2 during this $\mathrm{S}$ phase. It also remains an interesting question whether and how Mei2 acts on the $\mathrm{S}$ phase $\mathrm{CDK}$-cyclin complex to initiate premeiotic DNA synthesis.

\section{Implications from the $\mathbf{G}_{2}$-exit meiosis}

The meiotic program naturally starts from $G_{1}$ because activation of Mei2 is restricted to this phase of the cell cycle. However, in certain settings, meiosis appears to be induced from other cell cycle windows than $\mathrm{G}_{1}$ (Watanabe et al. 2001). In rumls cells, which have a very short $\mathrm{G}_{1}$ phase, meiosis can be induced from the $G_{2}$ window in three different ways to activate Mei2 artificially: (1) by inactivation of Pat1 kinase, (2) by ectopically expressing mei3 (see below), and (3) by ectopically expressing $m e i 2^{S A T A}$. It seems from these results that unphosphorylated Mei2 induces quiescence of cell proliferation rather than cell cycle arrest specific to $G_{1}$ phase 
(Figure 1). After the inhibition of cell proliferation, Mei2 appears to function to induce premeiotic DNA synthesis if cells are arrested in $\mathrm{G}_{1}$. However, if they are arrested in $G_{2}$, Mei2 appears to induce meiosis from $G_{2}$, which undergoes no DNA synthesis but only two rounds of nuclear division. If meiosis is induced from $\mathrm{G}_{2}$, DSC1-regulated genes required for both premitotic and premeiotic $\mathrm{S}$ phase, such as $c d c 18$ and $c d c 22$, fail to be expressed. Curiously, however, DSC1-regulated genes required only for premeiotic $\mathrm{S}$ phase, such as $\mathrm{rec} 8$, are expressed substantially, though the timing and the level of their expression may somewhat change (Watanabe et al. 2001). It may be too tempting to speculate that Mei2 can recognize in which window of the cell cycle the cells are and can start a meiotic program adjusted to the window. Alternatively, once the mei2-dependent growth inhibition is established, the state of the CDK activity and/or other cell cyclespecific features, such as the licensing state of replication origins, may autonomously set the start point of the meiotic program.

\section{Signal pathways linking extracellular cues to the commitment to meiosis}

Here we address which signaling cascades emanate from environmental stimuli to the activation of Mei2. This section provides an overview, and more detailed aspects will be discussed in the next four sections. The initiation of meiosis is regulated by two major signal transduction pathways in fission yeast, one responding to the nutritional conditions (Figure 3a) and the other to the mating pheromones (Figure 3b). Nutrient starvation results in transcriptional activation of the stell gene, through down-regulation of the activity of cAMP-dependent protein kinase (PKA) (Maeda et al. 1990, Sugimoto et al. 1991, Mochizuki \& Yamamoto 1992). Ste11, an HMG-type transcription factor, activates a number of genes required for mating and meiosis, including genes encoding mating pheromones and their receptors, mating-type genes (matl-Pc, matl-Pi, matl-Mc, and mat1-Mi), and mei2 (Sugimoto et al. 1991). Binding of the pheromone to its receptor results in activation of a MAPK cascade, which leads to the induction of additional genes required for the mating process. Pheromone communication and subsequent MAPK activation occur not only in haploid but also in diploid cells. In zygotes and diploid cells, $h^{+}$-specific Mat1-Pi and $h^{-}$-specific Mat1-Mc cooperatively induce the mei3 gene, which encodes an inhibitor of Pat1 kinase (McLeod \& Beach 1988, Li \& McLeod 1996, Van Heeckeren et al. 1998). The binding of Mei3 is thought to confer full inactivation of Pat1, thereby liberating Mei2 from the inhibitory phosphorylation (Watanabe et al. 1997). In cells under physiological conditions, complete inactivation of Pat1 is strictly dependent on expression of Mei3,

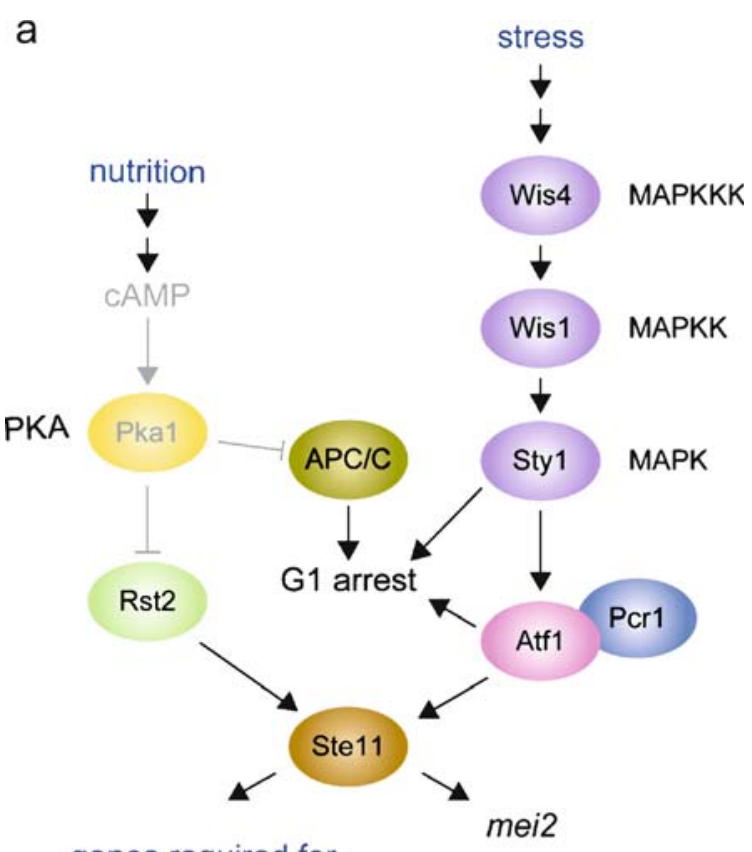

genes required for

\section{pheromone communication}

Figure 3. Signal transduction pathways that regulate the initiation of meiosis. (a) The cAMP-PKA pathway and the stress-responsive MAPK pathway mediate signals from external nutrition and stress, respectively, which ensure cell cycle arrest in $G_{1}$ and transcriptional activation of the stell gene under starved conditions. Ste11 activates a number of genes required for pheromone communication and induction of meiosis. (b) Binding of either $P$ or $M$ pheromone to its receptor leads to activation of the Byr2-Byr1-Spk1 pathway. Ste11 is again targeted by Spk1 to activate the pheromone-induced transcriptional program and to promote $\mathrm{G}_{1}$ arrest, both of which are prerequisites for the mating and meiotic process. Successful conjugation results in production of both $h^{+}$-specific Mat1-Pi and $h^{-}$-specific Mat1-Mc in a single cell, which cooperatively induce transcription of mei3. Mei3 inhibits Pat 1 kinase, and hence liberates Mei2 from the inhibitory phosphorylation. For simplification, many factors and interactions known to be involved in this process are omitted in this illustration. 


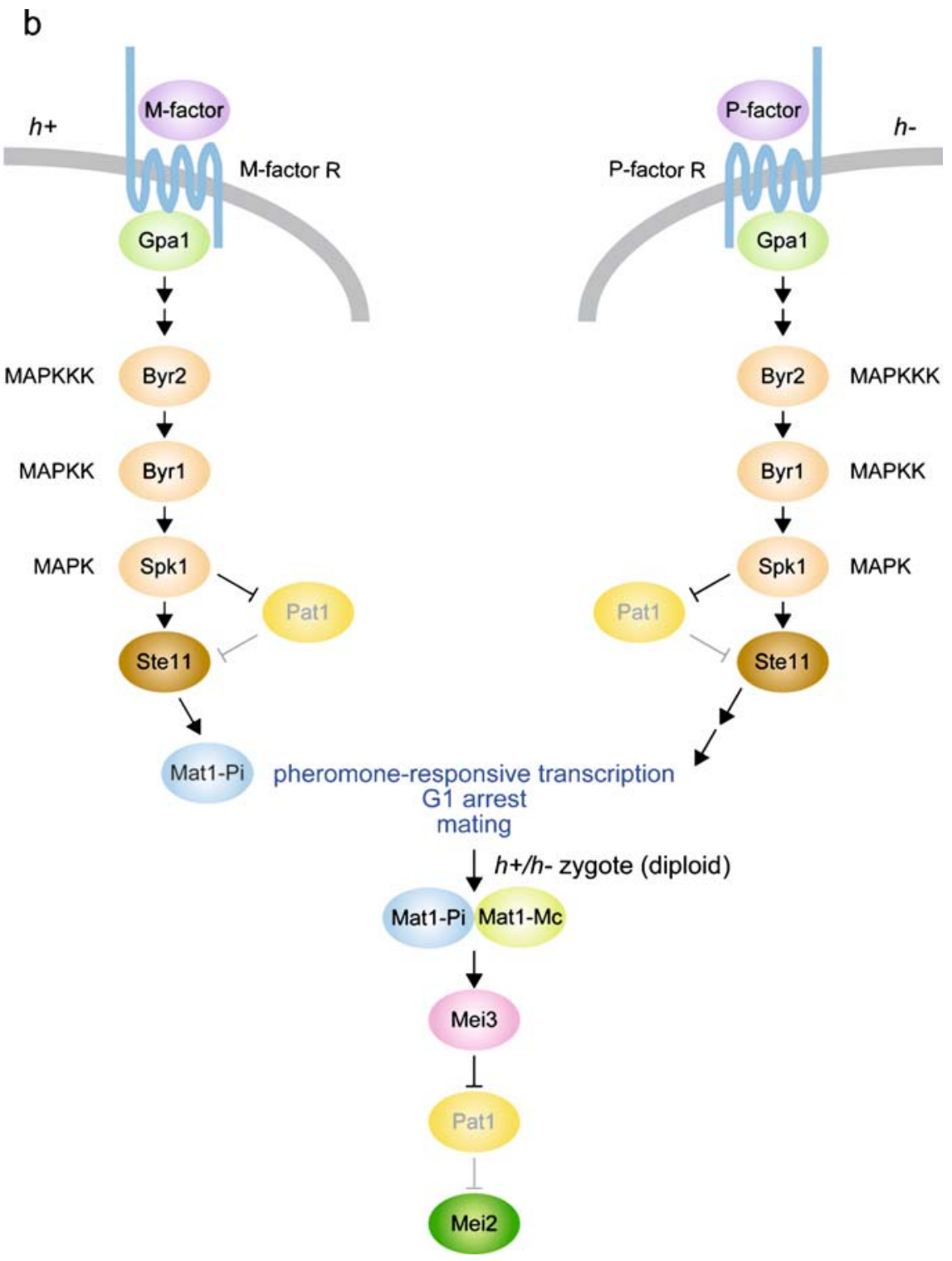

Figure 3. continued

which ensures that meiosis is induced only in diploid cells heterozygous for $P$ and $M$ mating-type genes.

\section{Nutritional starvation induces $\mathbf{G}_{\mathbf{1}}$ arrest and ste11 transcription}

The sexual differentiation process is triggered by reduced availability of nutrients in fission yeast, as in many microorganisms. Starvation, especially of nitrogen, promotes (1) arrest in the pre-Start $G_{1}$ phase, which is a prerequisite for sexual differentiation, and (2) transcriptional activation of the stell gene, which in turn activates transcription of a number of genes required for mating and meiosis (Figure 3a).

Starvation-induced pre-Start arrest requires downregulation of the CDK activity that drives the $G_{1} / S$ transition. It is necessary to inhibit both the Cdc2-Cig2 complex, which triggers timely S phase, and the Cdc2-Cdc13 complex, which can take over Cig2 function in its absence (Fisher \& Nurse 1996). 
Cig2 cyclin is thought to be a primary target for degradation in response to nutritional starvation (discussed in Nielsen 2004). Degradation of Cig2 delays $G_{1} / S$ transition, which leads to accumulation of Rum1. Rum1 directly inhibits the $\mathrm{Cdc} 2-\mathrm{Cdc} 13$ complex and promotes degradation of both $\mathrm{Cig} 2$ and Cdc13 via APC/C ${ }^{\text {ste9 }}$ (Correa-Bordes \& Nurse 1995). Two pathways, the cAMP-PKA pathway and the stress-responsive MAPK pathway, have been shown to link nutritional conditions with CDK regulation.

Nutrient starvation results in a reduced level of intracellular cAMP and cAMP-dependent protein kinase (PKA) activity (Maeda et al. 1990, Mochizuki $\&$ Yamamoto 1992). A reduction in the PKA activity relieves inhibitory phosphorylation of APC/C (Yamashita et al. 1996). This facilitates degradation of both the Cig2 and the Cdc13 cyclin.

Starvation of nutrients also leads to activation of the stress-responsive MAPK pathway (Wis4/Wak1 and Win1 MAPKKKs, Wis1 MAPKK, and Sty1/ Spc1 MAPK) (Samejima et al. 1997, 1998, Shieh et al. 1997, 1998, Shiozaki et al. 1997). A target of Sty1/Spc1 is a bZIP protein Atf1/Gad7, which constitutes a transcription-activating complex (Shiozaki \& Russell 1996, Wilkinson et al. 1996). The wisl, styl, and atfl genes are required for proper $\mathrm{G}_{1}$ arrest following nitrogen starvation (Warbrick \& Fantes 1991, Takeda et al. 1995, Kanoh et al. 1996, Shiozaki \& Russell 1996, Wilkinson et al. 1996). While the mechanism by which this stress pathway induces pre-Start $\mathrm{G}_{1}$ arrest is not yet known, the following scenario has been proposed based on the fact that nitrogen starvation speeds up mitosis, affecting $\mathrm{G}_{2} / \mathrm{M}$ transition through the stress pathway: Starvation-induced rapid mitosis results in smaller size of the newborn $G_{1}$ cells; To attain the critical size for entry to $S$ phase, the length of the $G_{1}$ window is prolonged; Consequently, Rum 1 protein accumulates in excess and down-regulates the CDK activity completely (Nielsen 2004). Consistent with this hypothesis, the atfl $\Delta$ strain can arrest at $\mathrm{G}_{1}$ if it carries the weel-50 mutation, which causes precocious $\mathrm{G}_{2} / \mathrm{M}$ transition (Kanoh et al. 1996).

Starvation-induced ste 11 expression is also mediated by the cAMP-PKA and the stress-responsive MAPK pathways. Rst2, a zinc-finger transcription factor, is a substrate of PKA and is inactive when phosphorylated by PKA (Kunitomo et al. 2000, Higuchi et al. 2002). The unphosphorylated form of Rst2 stimulates transcription of the stel1 gene. Atf1/
Gad7 and its binding partner Pcr1 are also apparently involved in the regulation of ste 11: Deletion of either atfl or pcrl reduces the level of stell expression considerably (Kanoh et al. 1996, Watanabe \& Yamamoto 1996). It remains to be determined whether the Atf1/Gad7-Pcr1 complex directly regulates the stell gene.

\section{Pheromone signaling}

The mating type of a fission yeast cell is determined by the DNA sequence at the matl locus. If it is $P$ (matl- $P$ ), the cell assumes the $h^{+}$mating type, and if it is $M$ (matl-M), the cell assumes the $h^{-}$mating type. Both matl- $P$ and matl-M consist of two divergently transcribed units, termed matl-Pc and matl-Pi, and matl-Mc and matl-Mi (Kelly et al. 1988). Whereas matl-Mc and matl-Pc are expressed in both mitotic and meiotic cells, though less intensively in the former, matl-Mi and matl-Pi are expressed only when the pheromone signaling is on (Kelly et al. 1988, Nielsen et al. 1992, Willer et al. 1995).

Ste11 activates transcription of genes required for pheromone communication (Sugimoto et al. 1991). The expression and the activity of Ste11 itself are apparently affected by both nitrogen starvation and pheromone signaling. This gives a somewhat complex aspect to overall gene regulation by Ste11, and certain genes targeted by Ste11 are known to be induced at various levels depending on the strength of these two signals (Aono et al. 1994, Sugiyama et al. 1994, Petersen et al. 1995). Ste11 enhances expression of matl-Pc in $h^{+}$cells and matl-Mc in $h^{-}$cells. These genes promote the production of mating pheromones and their receptors in a mating type-specific manner. The pheromone signal is mediated by Gpa1, a receptor-coupled G-protein, and a downstream MAPK module consisting of Byr2 (MAPKKK), Byr1 (MAPKK), and Spk1 (MAPK) (Obara et al. 1991, Gotoh et al. 1993, Neiman et al. 1993). Substrates phosphorylated by Spk1 are thought to cause the physiological effects of pheromone stimulation.

\section{Pheromone-induced transcription and $G_{1}$ arrest}

Many genes, including those targeted by Ste11, DSC1, or Mmi1, are transcriptionally up-regulated 
by pheromone stimulation (Chikashige et al. 2006, Harigaya et al. 2006, Mata \& Bahler 2006). However, connections between the pheromone pathway and the regulators of gene expression are mostly unclear, except for the relation between Spk1 and Ste11. It has been recently demonstrated that Ste11 is a target of MAP kinase Spk1 (Kjaerulff et al. 2005), but the physiological significance of this relation remains unknown.

Interestingly, transcription of some pheromonedependent genes, such as matl-Mi and fusl, takes place only in $G_{1}$ cells (Stern \& Nurse 1998). This may explain at least in part why cells do not conjugate when arrested in $\mathrm{G}_{2}$. A related observation is that nitrogen starvation fails to induce mei3 expression in $c d c 25-22$ or ruml $\Delta$ diploid cells, which have extended $G_{2}$ phase, suggesting a mechanism that restricts transcription of mei3 to $\mathrm{G}_{1}$ cells (Watanabe et al. 2001). This appears to ensure the start of the meiotic program from the $G_{1}$ window of the cell cycle. What then causes restriction of the pheromone-dependent transcription to $G_{1}$ ? The restriction could be due to cell cycle-specific downregulation of either the pheromone pathway or the downstream transcriptional apparatus. At least, however, nitrogen starvation-responsive activation of transcription by Ste11 seems to be independent of the cell cycle, because induction of Ste11 target genes, such as mei2, occurs normally in the nuc2-663 mutant, which is defective in $\mathrm{G}_{1}$ arrest (Kumada et al. 1995).

Experiments using synthetic pheromones have verified that the mating pheromone causes cell cycle arrest in $\mathrm{G}_{1}$ in fission yeast (Davey \& Nielsen 1994, Imai \& Yamamoto 1994). It is suggested that the pheromone blocks the onset of S phase by suppressing the Cdc2 kinase activity through Rum1 and APC/ C (Stern \& Nurse 1997, Stern \& Nurse 1998). Ste11, a target of the pheromone pathway, has been shown to contribute to this pheromone-induced $\mathrm{G}_{1}$ arrest (Kjaerulff et al. 2005).

\section{How is the nutritional status recognized?}

Whereas signals from the nutritional status, which are eventually transmitted to the master meiosisregulator Mei2, have been elucidated considerably, a fundamental question still remains unanswered: How do cells 'sense' the nutrition in molecular terms?
Recent studies on Tor2, one of the two TOR kinases in fission yeast, have provided an important clue (Alvarez \& Moreno 2006, Uritani et al. 2006, Matsuo et al. 2007, Weisman et al. 2007). Loss of tor 2 function apparently causes the same cellular responses as nitrogen starvation (i.e., entry to quiescence or conjugation in haploid cells and to meiosis in diploid cells). Thus, Tor2 appears to be central to the switch between cell growth and cell differentiation in response to the availability of nitrogen, and is likely to suppress other developmental fates than proliferation under rich nutrition. It is highly likely that down-regulation of Tor 2 activity is a physiological process that occurs in cells subjected to nitrogen starvation.

It has been shown that Tor2 forms a growthcontrolling TOR complex (TORC1), which is conserved from budding yeast to human cells and controls ribosome biogenesis (Alvarez \& Moreno 2006, Matsuo et al. 2007). One component of fission yeast TORC1 is the raptor ortholog Mip1, which has been shown to interact with Mei2 (Shinozaki-Yabana et al. 2000). Like mammalian cells, fission yeast has an ortholog of Rheb (Rhb1), which is a Ras superfamily GTPase, and orthologs of TSC1 and TSC2 (Tsc1 and Tsc2), which form a complex that acts as a GTPase activating protein (GAP) for Rheb. Rhb1 and the Tsc1-Tsc2 complex appear to function as upstream regulators of TORC1 in fission yeast (Matsumoto et al. 2002, van Slegtenhorst et al. 2004, Urano et al. 2005, 2007, Uritani et al. 2006, Matsuo et al. 2007, Weisman et al. 2007).

Unlike Tor2, Tor1 (the other TOR homolog) is nonessential for growth, but it is required for the entry to quiescence and sexual development (Kawai et al. 2001, Weisman \& Choder 2001, Matsuo et al. 2003). Tor 1 appears to be the major TOR kinase that forms TORC2 in fission yeast (Alvarez \& Moreno 2006, Matsuo et al. 2007).

\section{Conclusion and future directions}

The availability of nutrients is key for the mitosis-meiosis decision in fission yeast. Signals from nutrients converge on the master meiosis regulator Mei2. Although the entire suite of molecular functions that Mei2 exerts in order to provoke and orchestrate the meiotic program has not yet been unveiled, a Mei2 function essential for the initiation 
of meiosis I has now become apparent as suppression of the DSR-Mmi1 system. This system eliminates a subset of meiosis-specific transcripts during proliferation and hence blocks an untimely entry into meiosis. In future studies it will be intriguing to elucidate the remaining molecular functions of Mei2, such as that required to arrest cell proliferation or to elicit the meiotic cell cycle program following the exit from the mitotic cycle. Another important problem to be solved will be the molecular mechanism by which cells recognize nutritional conditions. Recent findings that fission yeast TORC1 apparently plays a pivotal role in this recognition seem to provide a promising prospect of solving this question.

\section{Acknowledgements}

We thank Akira Yamashita and Kayoko Tanaka for discussion during the preparation of this manuscript. We also thank Hiroshi Murakami for permission to refer to his unpublished results. Our recent studies cited in this review were supported by a Grant-in-Aid for Specially Promoted Research from the Ministry of Education, Culture, Sports, Science, and Technology (MEXT) of Japan to Y.M.

\section{References}

Alvarez B, Moreno S (2006) Fission yeast Tor2 promotes cell growth and represses cell differentiation. J Cell Sci 119: 4475-4485.

Aono T, Yanai H, Miki F, Davey J, Shimoda C (1994) Mating pheromone-induced expression of the matl-Pm gene of Schizosaccharomyces pombe: identification of signalling components and characterization of upstream controlling elements. Yeast 10: 757-770.

Averbeck N, Sunder S, Sample N, Wise JA, Leatherwood J (2005) Negative control contributes to an extensive program of meiotic splicing in fission yeast. Mol Cell 18: 491-498.

Baum B, Wuarin J, Nurse P (1997) Control of S-phase periodic transcription in the fission yeast mitotic cycle. EMBO $J \mathbf{1 6}$ : 4676-4688.

Beach D, Rodgers L, Gould J (1985) ranl $^{+}$controls the transition from mitotic division to meiosis in fission yeast. Curr Genet 10: 297-311.

Blanco MA, Sanchez-Diaz A, de Prada JM, Moreno S (2000) $\mathrm{APC}^{\text {ste9/srw1 }}$ promotes degradation of mitotic cyclins in $\mathrm{G}_{1}$ and is inhibited by cdc2 phosphorylation. EMBO J 19: 3945-3955.

Borgne A, Murakami H, Ayte J, Nurse P (2002) The $G_{1} / S$ cyclin Cig2p during meiosis in fission yeast. Mol Biol Cell 13: 2080-2090.
Bueno A, Russell P (1993) Two fission yeast B-type cyclins, Cig2 and $\mathrm{Cdc} 13$, have different functions in mitosis. Mol Cell Biol 13: 2286-2297.

Caligiuri M, Beach D (1993) Sct1 functions in partnership with Cdc10 in a transcription complex that activates cell cycle START and inhibits differentiation. Cell 72: 607-619.

Chikashige Y, Tsutsumi C, Yamane M, Okamasa K, Haraguchi T, Hiraoka Y (2006) Meiotic proteins bqt1 and bqt2 tether telomeres to form the bouquet arrangement of chromosomes. Cell 125: 59-69.

Connolly T, Beach D (1994) Interaction between the Cig1 and Cig2 B-type cyclins in the fission yeast cell cycle. Mol Cell Biol 14: 768-776.

Correa-Bordes J, Nurse P (1995) p25 ${ }^{\text {rum } 1}$ orders S phase and mitosis by acting as an inhibitor of the $\mathrm{p} 34^{\mathrm{cdc} 2}$ mitotic kinase. Cell 83: 1001-1009.

Costello G, Rodgers L, Beach D (1986) Fission yeast enters the stationary phase $\mathrm{G} 0$ state from either mitotic $\mathrm{G}_{1}$ or $\mathrm{G}_{2}$. Curr Genet 11: 119-125.

Cunliffe L, White S, McInerny CJ (2004) DSC1-MCB regulation of meiotic transcription in Schizosaccharomyces pombe. Mol Genet Genomics 271: 60-71.

Davey J, Nielsen O (1994) Mutations in cyrl and patl reveal pheromone-induced $\mathrm{G}_{1}$ arrest in the fission yeast Schizosaccharomyces pombe. Curr Genet 26: 105-112.

Fernandez-Sarabia MJ, McInerny C, Harris P, Gordon C, Fantes P (1993) The cell cycle genes $c d c 22^{+}$and $s u c 22^{+}$of the fission yeast Schizosaccharomyces pombe encode the large and small subunits of ribonucleotide reductase. Mol Gen Genet 238: 241-251.

Fisher DL, Nurse P (1996) A single fission yeast mitotic cyclin B $\mathrm{p} 34^{\mathrm{cdc} 2}$ kinase promotes both S-phase and mitosis in the absence of $\mathrm{G}_{1}$ cyclins. EMBO $J$ 15: 850-860.

Fox ME, Smith GR (1998) Control of meiotic recombination in Schizosaccharomyces pombe. Prog Nucleic Acid Res Mol Biol 61: $345-378$.

Gotoh Y, Nishida E, Shimanuki M, Toda T, Imai Y, Yamamoto M (1993) Schizosaccharomyces pombe Spk1 is a tyrosinephosphorylated protein functionally related to Xenopus mitogen-activated protein kinase. Mol Cell Biol 13: 6427-6434.

Harigaya Y, Tanaka H, Yamanaka S, et al. (2006) Selective elimination of messenger RNA prevents an incidence of untimely meiosis. Nature 442: 45-50.

Higuchi T, Watanabe Y, Yamamoto M (2002) Protein kinase A regulates sexual development and gluconeogenesis through phosphorylation of the $\mathrm{Zn}$ finger transcriptional activator Rst2p in fission yeast. Mol Cell Biol 22: 1-11.

Hofmann JF, Beach D (1994) cdt1 is an essential target of the Cdc10/Sct1 transcription factor: requirement for DNA replication and inhibition of mitosis. EMBO J 13: 425-434.

Horie S, Watanabe Y, Tanaka K, et al. (1998) The Schizosaccharomyces pombe mei4 $^{+}$gene encodes a meiosis-specific transcription factor containing a forkhead DNA-binding domain. Mol Cell Biol 18: 2118-2129.

Houseley J, LaCava J, Tollervey D (2006) RNA-quality control by the exosome. Nat Rev Mol Cell Biol 7: 529-539.

Iino Y, Yamamoto M (1985) Mutants of Shizosaccharomyces pombe which sporulate in the haploid state. Mol Gen Genet 198: 416. 
Iino Y, Hiramine Y, Yamamoto M (1995) The role of $c d c 2$ and other genes in meiosis in Schizosaccharomyces pombe. Genetics 140: $1235-1245$.

Imai Y, Yamamoto M (1994) The fission yeast mating pheromone P-factor: its molecular structure, gene structure, and ability to induce gene expression and $\mathrm{G}_{1}$ arrest in the mating partner. Genes Dev 8: 328-338.

Izawa D, Goto M, Yamashita A, Yamano H, Yamamoto M (2005) Fission yeast Mes1p ensures the onset of meiosis II by blocking degradation of cyclin Cdc13p. Nature 434: 529-533.

Kanoh J, Watanabe Y, Ohsugi M, Ino Y, Yamamoto M (1996) Schizosaccharomyces pombe gad $7^{+}$encodes a phosphoprotein with a bZIP domain, which is required for proper $\mathrm{G}_{1}$ arrest and gene expression under nitrogen starvation. Genes Cells 1: 391-408.

Kawai M, Nakashima A, Ueno M, et al. (2001) Fission yeast tor1 functions in response to various stresses including nitrogen starvation, high osmolarity, and high temperature. Curr Genet 39: $166-174$.

Keeney S, Giroux CN, Kleckner N (1997) Meiosis-specific DNA double-strand breaks are catalyzed by Spo11, a member of a widely conserved protein family. Cell 88: $375-384$.

Kelly M, Burke J, Smith M, Klar A, Beach D (1988) Four matingtype genes control sexual differentiation in the fission yeast. EMBO J 7: 1537-1547.

Kelly TJ, Martin GS, Forsburg SL, Stephen RJ, Russo A, Nurse P (1993) The fission yeast $c d c 18^{+}$gene product couples $S$ phase to START and mitosis. Cell 74: 371-382.

Kitamura K, Maekawa H, Shimoda C (1998) Fission yeast Ste9, a homolog of Hct1/Cdh1 and Fizzy-related, is a novel negative regulator of cell cycle progression during $\mathrm{G}_{1}$-phase. Mol Biol Cell 9: 1065-1080.

Kitamura K, Katayama S, Dhut S, et al. (2001) Phosphorylation of Mei2 and Ste11 by Pat1 kinase inhibits sexual differentiation via ubiquitin proteolysis and 14-3-3 protein in fission yeast. Dev Cell 1: 389-399.

Kjaerulff S, Lautrup-Larsen I, Truelsen S, Pedersen M, Nielsen O (2005) Constitutive activation of the fission yeast pheromoneresponsive pathway induces ectopic meiosis and reveals ste 11 as a mitogen-activated protein kinase target. Mol Cell Biol 25: 2045-2059.

Kumada K, Su S, Yanagida M, Toda T (1995) Fission yeast TPRfamily protein nuc2 is required for $\mathrm{G}_{1}$-arrest upon nitrogen starvation and is an inhibitor of septum formation. J Cell Sci 108 (Pt 3): 895-905.

Kunitomo H, Higuchi T, Iino Y, Yamamoto M (2000) A zincfinger protein, Rst2p, regulates transcription of the fission yeast ste $\mathrm{Il}^{+}$gene, which encodes a pivotal transcription factor for sexual development. Mol Biol Cell 11: 3205-3217.

Li P, McLeod M (1996) Molecular mimicry in development: identification of $\mathrm{ste} \mathrm{I}^{+}$as a substrate and $\mathrm{mei}^{+}$as a pseudosubstrate inhibitor of ranl $^{+}$kinase. Cell 87: 869-880.

Lowndes NF, McInerny CJ, Johnson AL, Fantes PA, Johnston LH (1992) Control of DNA synthesis genes in fission yeast by the cell-cycle gene $c d c 10^{+}$. Nature 355: 449-453.

Lundgren K, Walworth N, Booher R, Dembski M, Kirschner M, Beach D (1991) mik1 and weel cooperate in the inhibitory tyrosine phosphorylation of cdc2. Cell 64: 1111-1122.
MacNeill SA, Nurse P (1997) Cell cycle control in fission yeast. In: Pringle J, Broach $\mathrm{J}$, Jones E, eds. The Molecular and Cellular Biology of the Yeast Saccahromyces. Cold Spring Harbor, NY: Cold Spring Harbor Laboratory Press.

Maeda T, Mochizuki N, Yamamoto M (1990) Adenylyl cyclase is dispensable for vegetative cell growth in the fission yeast Schizosaccharomyces pombe. Proc Natl Acad Sci USA 87: 7814-7818.

Malapeira J, Moldon A, Hidalgo E, Smith GR, Nurse P, Ayte J (2005) A meiosis-specific cyclin regulated by splicing is required for proper progression through meiosis. Mol Cell Biol 25: $6330-6337$.

Mata J, Bahler J (2006) Global roles of Ste11p, cell type, and pheromone in the control of gene expression during early sexual differentiation in fission yeast. Proc Natl Acad Sci USA 103: $15517-15522$

Mata J, Lyne R, Burns G, Bahler J (2002) The transcriptional program of meiosis and sporulation in fission yeast. Nat Genet 32: 143-147.

Matsumoto S, Bandyopadhyay A, Kwiatkowski DJ, Maitra U, Matsumoto T (2002) Role of the Tsc1-Tsc2 complex in signaling and transport across the cell membrane in the fission yeast Schizosaccharomyces pombe. Genetics 161: 1053-1063.

Matsuo T, Kubo Y, Watanabe Y, Yamamoto M (2003) Schizosaccharomyces pombe AGC family kinase Gad8p forms a conserved signaling module with TOR and PDK1-like kinases. EMBO J 22: 3073-3083.

Matsuo T, Otsubo Y, Urano J, Tamanoi F, Yamamoto M (2007) Loss of the TOR kinase Tor2 mimics nitrogen starvation and activates the sexual development pathway in fission yeast. Mol Cell Biol 27: 3154-3164.

McLeod M, Beach D (1988) A specific inhibitor of the ranl ${ }^{+}$ protein kinase regulates entry into meiosis in Schizosaccharomyces pombe. Nature 332: 509-514.

Millar JB, McGowan CH, Lenaers G, Jones R, Russell P (1991) $\mathrm{p} 80^{\text {cdc25 }}$ mitotic inducer is the tyrosine phosphatase that activates $\mathrm{p} 34^{\mathrm{cdc} 2}$ kinase in fission yeast. EMBO J 10: 4301-4309.

Miyamoto M, Tanaka K, Okayama H (1994) res2 ${ }^{+}$, a new member of the $c d c 10^{+} / S W I 4$ family, controls the 'start' of mitotic and meiotic cycles in fission yeast. EMBO J 13: 1873-1880.

Mochizuki N, Yamamoto M (1992) Reduction in the intracellular cAMP level triggers initiation of sexual development in fission yeast. Mol Gen Genet 233: 17-24.

Moser BA, Russell P (2000) Cell cycle regulation in Schizosaccharomyces pombe. Curr Opin Microbiol 3: 631-636.

Nakashima N, Tanaka K, Sturm S, Okayama H (1995) Fission yeast Rep2 is a putative transcriptional activator subunit for the cell cycle 'start' function of Res2-Cdc10. EMBO J 14: 4794-4802.

Neiman AM, Stevenson BJ, Xu HP, et al. (1993) Functional homology of protein kinases required for sexual differentiation in Schizosaccharomyces pombe and Saccharomyces cerevisiae suggests a conserved signal transduction module in eukaryotic organisms. Mol Biol Cell 4: 107-120.

Nielsen O (2004) Mating-type control and differentiation. In: Egel $\mathrm{R}$, ed. The Moleular Biology of Schizosaccharomyces pombe. Berlin, Heidelberg: Springer, pp. 281-296. 
Nielsen O, Davey J, Egel R (1992) The ras1 function of Schizosaccharomyces pombe mediates pheromone-induced transcription. EMBO J 11: 1391-1395.

Nurse P (1985) Mutants of the Shizosaccharomyces pombe which alter the shift between cell proliferation and sporulation. Mol Gen Genet 198: 497.

Obara T, Nakafuku M, Yamamoto M, Kaziro Y (1991) Isolation and characterization of a gene encoding a G-protein $\alpha$ subunit from Schizosaccharomyces pombe: involvement in mating and sporulation pathways. Proc Natl Acad Sci USA 88: 5877-5881.

Obara-Ishihara T, Okayama H (1994) A B-type cyclin negatively regulates conjugation via interacting with cell cycle 'start' genes in fission yeast. EMBO J 13: 1863-1872.

Petersen J, Weilguny D, Egel R, Nielsen O (1995) Characterization of fus 1 of Schizosaccharomyces pombe: a developmentally controlled function needed for conjugation. Mol Cell Biol 15: 3697-3707.

Samejima I, Mackie S, Fantes PA (1997) Multiple modes of activation of the stress-responsive MAP kinase pathway in fission yeast. EMBO J 16: 6162-6170.

Samejima I, Mackie S, Warbrick E, Weisman R, Fantes PA (1998) The fission yeast mitotic regulator $w i n 1^{+}$encodes an MAP kinase kinase kinase that phosphorylates and activates Wis1 MAP kinase kinase in response to high osmolarity. Mol Biol Cell 9: 2325-2335.

Sato M, Shinozaki-Yabana S, Yamashita A, Watanabe Y, Yamamoto M (2001) The fission yeast meiotic regulator Mei2p undergoes nucleocytoplasmic shuttling. FEBS Lett 499: 251-255.

Sato M, Watanabe Y, Akiyoshi Y, Yamamoto M (2002) 14-3-3 protein interferes with the binding of RNA to the phosphorylated form of fission yeast meiotic regulator Mei2p. Curr Biol 12: 141-145.

Shieh JC, Wilkinson MG, Buck V, Morgan BA, Makino K, Millar JB (1997) The Mcs4 response regulator coordinately controls the stress-activated Wak1-Wis1-Sty1 MAP kinase pathway and fission yeast cell cycle. Genes Dev 11: 1008-1022.

Shieh JC, Wilkinson MG, Millar JB (1998) The Win1 mitotic regulator is a component of the fission yeast stress-activated Sty1 MAPK pathway. Mol Biol Cell 9: 311-322.

Shimada T, Yamashita A, Yamamoto M (2003) The fission yeast meiotic regulator Mei2p forms a dot structure in the horse-tail nucleus in association with the sme 2 locus on chromosome II. Mol Biol Cell 14: 2461-2469.

Shinozaki-Yabana S, Watanabe Y, Yamamoto M (2000) Novel WD-repeat protein Mip1p facilitates function of the meiotic regulator Mei2p in fission yeast. Mol Cell Biol 20: 1234-1242.

Shiozaki K, Russell P (1996) Conjugation, meiosis, and the osmotic stress response are regulated by Spc1 kinase through Atf1 transcription factor in fission yeast. Genes Dev 10: 2276-2288.

Shiozaki K, Shiozaki M, Russell P (1997) Mcs4 mitotic catastrophe suppressor regulates the fission yeast cell cycle through the Wik1-Wis1-Spc1 kinase cascade. Mol Biol Cell 8: 409-419.

Stern B, Nurse P (1997) Fission yeast pheromone blocks S-phase by inhibiting the $\mathrm{G}_{1}$ cyclin $\mathrm{B}-\mathrm{p} 34^{\text {cdc2 }}$ kinase. EMBO $J$ 16: 534-544.

Stern B, Nurse P (1998) Cyclin B proteolysis and the cyclindependent kinase inhibitor rum $1 p$ are required for pheromoneinduced $\mathrm{G}_{1}$ arrest in fission yeast. Mol Biol Cell 9: 1309-1321.
Stoilov P, Rafalska I, Stamm S (2002) YTH: a new domain in nuclear proteins. Trends Biochem Sci 27: 495-497.

Sugimoto A, Iino Y, Maeda T, Watanabe Y, Yamamoto M (1991) Schizosaccharomyces pombe stel $1^{+}$encodes a transcription factor with an HMG motif that is a critical regulator of sexual development. Genes Dev 5: 1990-1999.

Sugiyama A, Tanaka K, Okazaki K, Nojima H, Okayama H (1994) A zinc finger protein controls the onset of premeiotic DNA synthesis of fission yeast in a Mei2-independent cascade. EMBO J 13: 1881-1887.

Takeda T, Toda T, Kominami K, Kohnosu A, Yanagida M, Jones $\mathrm{N}$ (1995) Schizosaccharomyces pombe atfl ${ }^{+}$encodes a transcription factor required for sexual development and entry into stationary phase. EMBO J 14: 6193-6208.

Tanaka K, Okazaki K, Okazaki N, et al. (1992) A new cdc gene required for $\mathrm{S}$ phase entry of Schizosaccharomyces pombe encodes a protein similar to the $c d c 10^{+}$and SWI4 gene products. EMBO J 11: 4923-4932.

Urano J, Comiso MJ, Guo L, et al. (2005) Identification of novel single amino acid changes that result in hyperactivation of the unique GTPase, Rheb, in fission yeast. Mol Microbiol 58: 1074-1086.

Urano J, Sato T, Matsuo T, Otsubo Y, Yamamoto M, Tamanoi F (2007) Point mutations in TOR confer Rheb-independent growth in fission yeast and nutrient-independent mammalian TOR signaling in mammalian cells. Proc Natl Acad Sci USA 104: 3514-3519.

Uritani M, Hidaka H, Hotta Y, Ueno M, Ushimaru T, Toda T (2006) Fission yeast Tor2 links nitrogen signals to cell proliferation and acts downstream of the Rheb GTPase. Genes Cells 11: 1367-1379.

Van Heeckeren WJ, Dorris DR, Struhl K (1998) The mating-type proteins of fission yeast induce meiosis by directly activating mei3 transcription. Mol Cell Biol 18: 7317-7326.

van Slegtenhorst M, Carr E, Stoyanova R, Kruger WD, Henske EP (2004) $\mathrm{Tscl}^{+}$and $t s c 2^{+}$regulate arginine uptake and metabolism in Schizosaccharomyces pombe. J Biol Chem 279: 12706-12713.

Warbrick E, Fantes PA (1991) The wis1 protein kinase is a dosagedependent regulator of mitosis in Schizosaccharomyces pombe. EMBO J 10: 4291-4299.

Watanabe Y, Nurse P (1999) Cohesin Rec8 is required for reductional chromosome segregation at meiosis. Nature 400: 461-464.

Watanabe Y, Yamamoto M (1994) S. pombe mei2 $2^{+}$encodes an RNA-binding protein essential for premeiotic DNA synthesis and meiosis I, which cooperates with a novel RNA species meiRNA. Cell 78: 487-498.

Watanabe Y, Yamamoto M (1996) Schizosaccharomyces pombe pcrl ${ }^{+}$encodes a CREB/ATF protein involved in regulation of gene expression for sexual development. Mol Cell Biol 16: 704-711.

Watanabe Y, Shinozaki-Yabana S, Chikashige Y, Hiraoka Y, Yamamoto M (1997) Phosphorylation of RNA-binding protein controls cell cycle switch from mitotic to meiotic in fission yeast. Nature 386: 187-190.

Watanabe Y, Yokobayashi S, Yamamoto M, Nurse P (2001) Pre-meiotic $\mathrm{S}$ phase is linked to reductional chromosome segregation and recombination. Nature 409: 359-363. 
Weisman R, Choder M (2001) The fission yeast TOR homolog, tor $1^{+}$, is required for the response to starvation and other stresses via a conserved serine. J Biol Chem 276: 7027-7032.

Weisman R, Roitburg I, Schonburn M, Harari R, Kupiec M (2007) Opposite effects of Tor1 and Tor2 on nitrogen starvation responses in fission yeast. Genetics 175: 1153-1162.

White S, Khaliq F, Sotiriou S, McInerny CJ (2001) The role of DSC1 components $c d c 10^{+}$, repl $^{+}$and rep $2^{+}$in $\mathrm{MCB}$ gene transcription at the mitotic $\mathrm{G}_{1}-\mathrm{S}$ boundary in fission yeast. Curr Genet 40: 251-259.

Whitehall S, Stacey P, Dawson K, Jones N (1999) Cell cycleregulated transcription in fission yeast: $\mathrm{Cdc} 10-\mathrm{Res}$ protein interactions during the cell cycle and domains required for regulated transcription. Mol Biol Cell 10: 3705-3715.

Wilkinson MG, Samuels M, Takeda T, et al. (1996) The Atf1 transcription factor is a target for the Sty1 stress-activated MAP kinase pathway in fission yeast. Genes Dev 10: 2289-2301.

Willer M, Hoffmann L, Styrkarsdottir U, Egel R, Davey J, Nielsen O (1995) Two-step activation of meiosis by the mat1 locus in Schizosaccharomyces pombe. Mol Cell Biol 15: 4964-4970.

Yamaguchi S, Murakami H, Okayama H (1997) A WD repeat protein controls the cell cycle and differentiation by negatively regulating Cdc2/B-type cyclin complexes. Mol Biol Cell 8: 2475-2486.

Yamamoto M (1996) The molecular control mechanisms of meiosis in fission yeast. Trends Biochem Sci 21: 18-22.

Yamamoto M (2004) Initiation of Meiosis. In: Egel R, ed. The Molecular Biology of Schizosaccharomyces pombe. Berlin, Heidelberg: Springer, pp 297-310.

Yamamoto M, Imai Y, Watanabe Y (1997) Mating and sporulation in Shizosaccharomyces pombe. In: Pringle J, Broach J, Jones E eds. The Molecular and Cellular Biology of the Yeast Saccahromyces. Cold Spring Harbor, NY: Cold Spring Harbor Laboratory Press, pp 1037-1106.

Yamashita A, Watanabe Y, Nukina N, Yamamoto M (1998) RNAassisted nuclear transport of the meiotic regulator Mei2p in fission yeast. Cell 95: 115-123.

Yamashita YM, Nakaseko Y, Samejima I, et al. (1996) 20S cyclosome complex formation and proteolytic activity inhibited by the cAMP/PKA pathway. Nature 384: 276-279.

Zhu Y, Takeda T, Nasmyth K, Jones N (1994) pct1 ${ }^{+}$, which encodes a new DNA-binding partner of $\mathrm{p} 85^{\mathrm{cdc} 10}$, is required for meiosis in the fission yeast Schizosaccharomyces pombe. Genes Dev 8: 885-898. 